\title{
The double shunt technique as a bridge to heart transplantation in a patient with pulmonary atresia with intact septum and right ventricular-dependent coronary circulation
}

Sameh M. Said, MBBCh, MD, FACS, ${ }^{a}$ Gamal Marey, MD, ${ }^{\mathrm{a}}$ Ryan Greene, MD, ${ }^{\mathrm{b}}$ Massimo Griselli, MD, ${ }^{\mathrm{a}}$ Gurumurthy Hiremath, MD, ${ }^{\mathrm{b}}$ Varun Aggarwal, MBBS, ${ }^{\mathrm{b}}$ and Elizabeth Braunlin, $\mathrm{MD}, \mathrm{PhD},{ }^{\mathrm{b}}$ Minneapolis, Minn

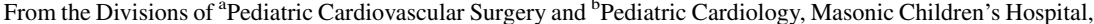
University of Minnesota, Minneapolis, Minn.

Disclosures: Dr Said is a consultant for Cryolife. All other authors reported no conflicts of interest.

The Journal policy requires editors and reviewers to disclose conflicts of interest and to decline handling or reviewing manuscripts for which they may have a conflict of interest. The editors and reviewers of this article have no conflicts of interest.

Received for publication Jan 11, 2021; accepted for publication Jan 11, 2021; available ahead of print Jan 19, 2021.

Address for reprints: Sameh M. Said, MBBCh, MD, FACS, Division of Pediatric Cardiovascular Surgery, Masonic Children's Hospital, University of Minnesota, 2450 Riverside Ave, S, East Building, MB 539, Minneapolis, MN 55454 (E-mail: ssaid@umn.edu).

JTCVS Techniques 2021;7:216-21

2666-2507

Copyright (c) 2021 The Authors. Published by Elsevier Inc. on behalf of The American Association for Thoracic Surgery. This is an open access article under the CC BY-NC-ND license (http://creativecommons.org/licenses/bync-nd/4.0/).

https://doi.org/10.1016/j.xjtc.2021.01.018
}

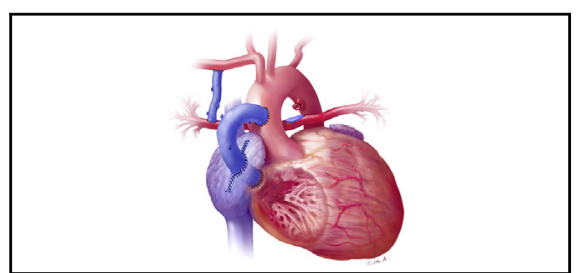

Aorto-tricuspid-right ventricular and systemic-topulmonary artery shunts in PA/IVS/RVDCC.

\section{CENTRAL MESSAGE \\ The double shunt technique (systemic-to-pulmonary artery, and aorto-tricuspid-right ven- tricular shunts), is a good initial palliation in PA/IVS and right ventricle-dependent coronary circulation.}

See Commentaries on pages 222 and 224 .
Pulmonary atresia with intact ventricular septum (PA/IVS) is a rare congenital heart defect with a broad spectrum that is associated with varying degrees of tricuspid valve (TV) and right ventricular (RV) hypoplasia and a wide range of coronary abnormalities. The most extreme form is right ventricular-dependent coronary circulation (RVDCC), which is estimated to occur in $3 \%$ to $34 \%$ of these cases. We present a neonate with PA/IVS, RVDCC, and bilateral coronary ostial atresia (COA) who was bridged by a "double shunt" strategy until heart transplantation.

\section{CASE REPORT}

A $2.6-\mathrm{kg}$ female neonate, born at 39 weeks' gestation, had a fetal diagnosis of PA/IVS with severe hypoplasia of the TV and the RV and a large tortuous ductus arteriosus. Prostaglandin infusion was initiated after birth and postnatal echocardiography revealed bilateral COA, and a TV annulus measuring $2.4 \mathrm{~mm}$ ( $z$ score, -5.7 ) with moderate TV regurgitation. Cardiac catheterization was performed to confirm the presence of RV-dependent coronary circulation and the COA (Figure 1, A). Right ventriculogram showed an epicardial network of coronary arteries with no well-defined major coronaries (Figure 1, B). Management options discussed included continuation of prostaglandin therapy and listing for transplantation, modified BlalockTaussig (MBTS) shunt, ductal (patent ductal arteriosus) stenting and listing for transplant, combined MBTS and aorto-RV shunt, and palliative care. No survival has been reported without transplantation in this subgroup of patients with bilateral $\mathrm{COA}^{2}$

Significant ST changes with continued elevation in her cardiac biomarkers were concerning while she was maintained on prostaglandin therapy. The decision was made to proceed with early listing for transplantation in addition to performing a combined MBTS and aorto-TV-RV shunt to increase coronary flow (Figure 2).

On day of life 6, and through a median sternotomy (Video 1), cardiopulmonary bypass was initiated via aortic and right atrial (RA) cannulation. Both branch pulmonary arteries were isolated with vessel loops, and the right side 


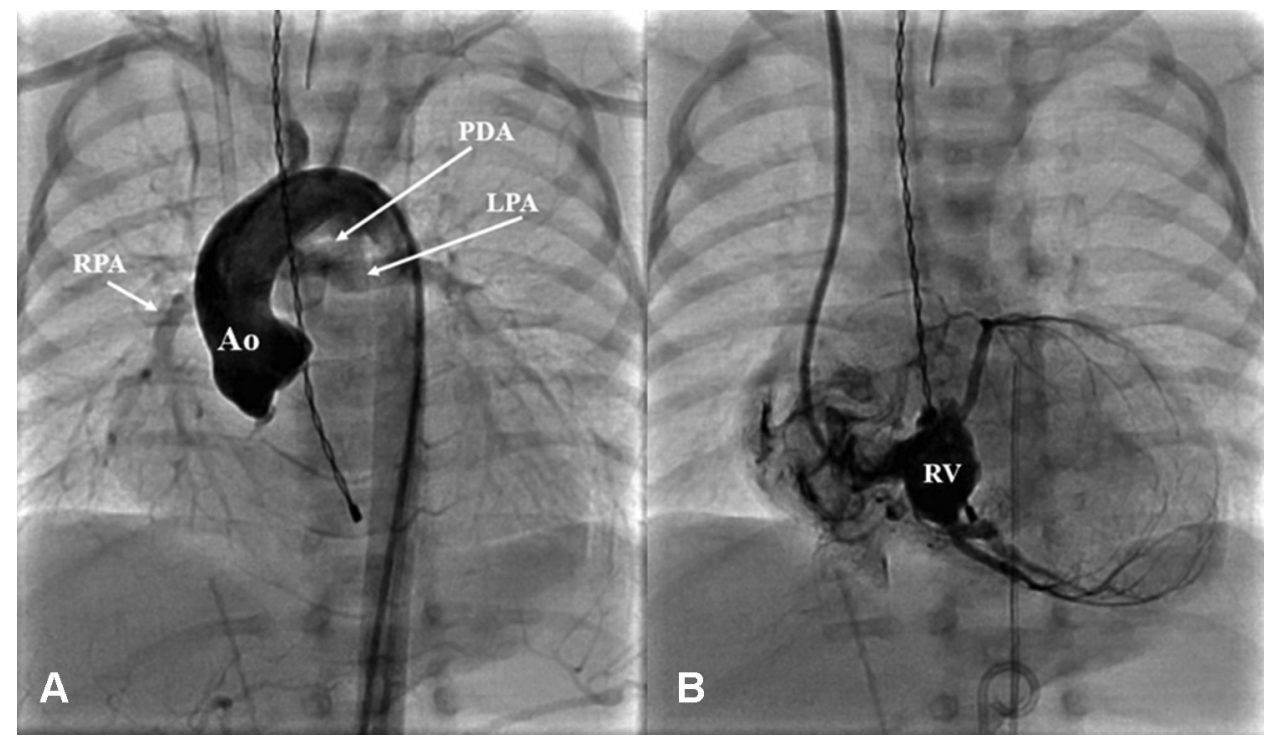

FIGURE 1. Preoperative cardiac catheterization. A, Aortogram showing bilateral coronary ostial atresia and ductal dependent pulmonary circulation. B, Right ventriculogram demonstrating the abnormal right ventricular-to-coronary communications. $R P A$, Right pulmonary artery; $A o$, aorta; $P D A$, patent ductus arteriosus; $L P A$, left pulmonary artery; $R V$, right ventricle.

of the heart was kept full until it was successfully arrested with cardioplegia that was administered by direct injection into the RV (Figure 3, A). The single RA cannula was converted to bicaval cannulation with both cavae snared. An aortic crossclamp was applied, and through a right atriotomy, the TV leaflets were excised (Figure 3, B). A 3-mm dilator fitted perfectly inside the TV orifice and the distal

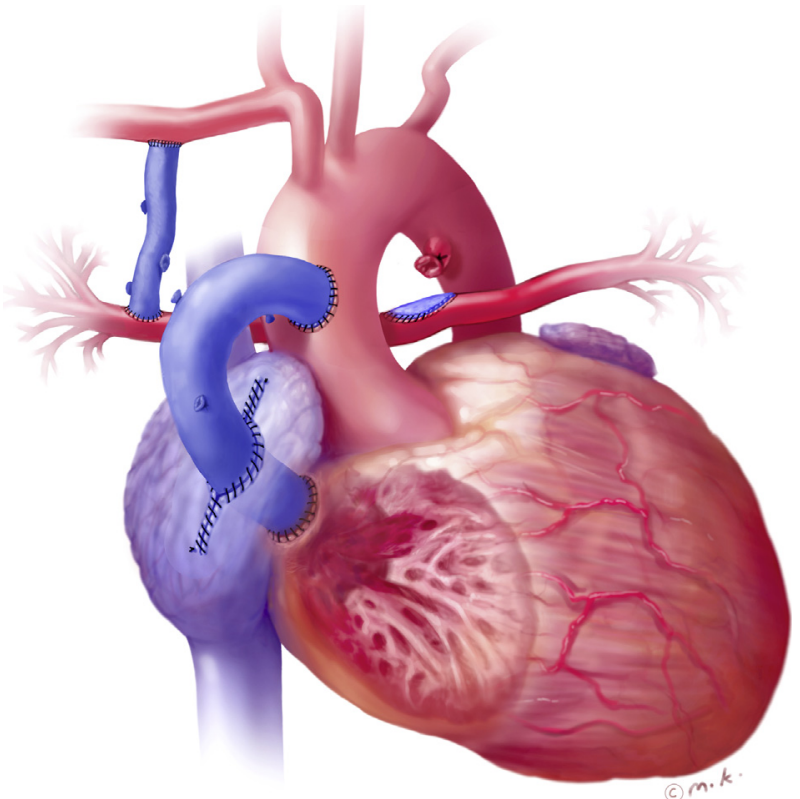

FIGURE 2. Final appearance of the double shunts with a right modified Blalock-Taussig and an aorto-tricuspid-right ventricular shunt using cryopreserved saphenous vein homografts. anastomosis of a 4-mm (external diameter) cryopreserved saphenous vein graft was constructed to the TV annulus using running 6-0 polypropylene suture (Figure 3, C, inset). The proximal anastomosis was constructed to the ascending aorta. The crossclamp was removed, followed by creation of a MBTS using another 4-mm cryopreserved saphenous vein (Figure 3,C). She was weaned off cardiopulmonary bypass with good ventricular function, and intraoperative fluorescence confirmed patency of the shunt (Video 2). However, she required temporary support with extracorporeal membrane oxygenation (ECMO) that was initiated at the end of the procedure due to high pressor requirements and lung injury from cardiopulmonary bypass. At that time, the MBTS was partially clipped to minimize coronary

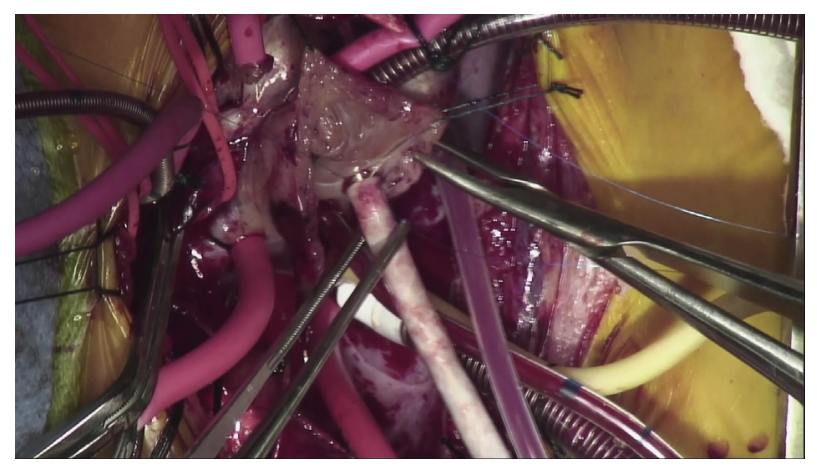

VIDEO 1. Operative video showing the technical steps in performing the double shunt technique in a 6-day-old neonate with pulmonary atresia intact septum and right ventricular-dependent coronary circulation. Video available at: https://www.jtcvs.org/article/S2666-2507(21)00084-5/ fulltext. 

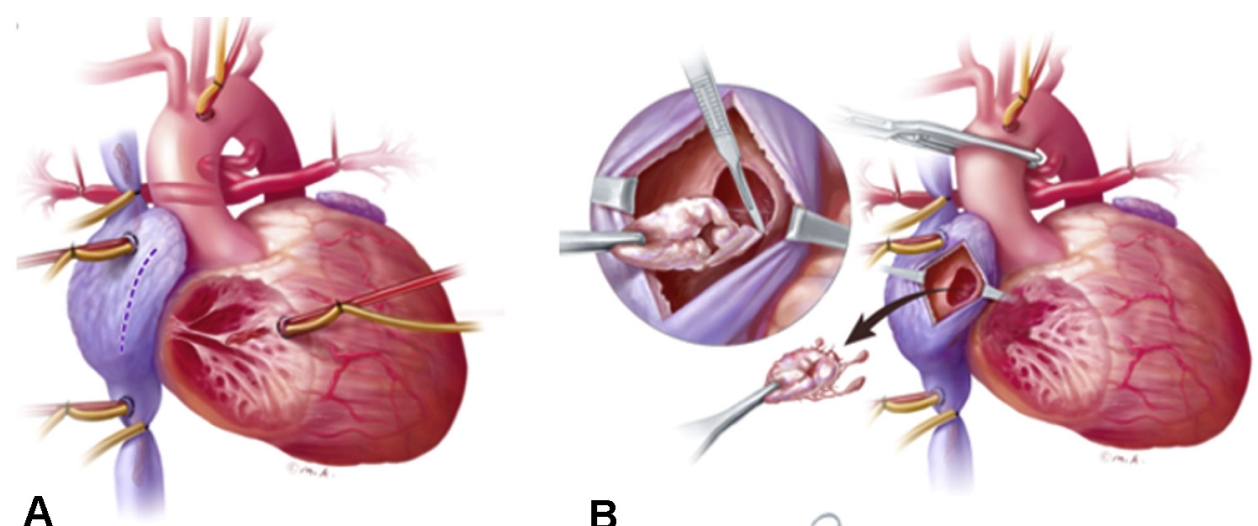

A

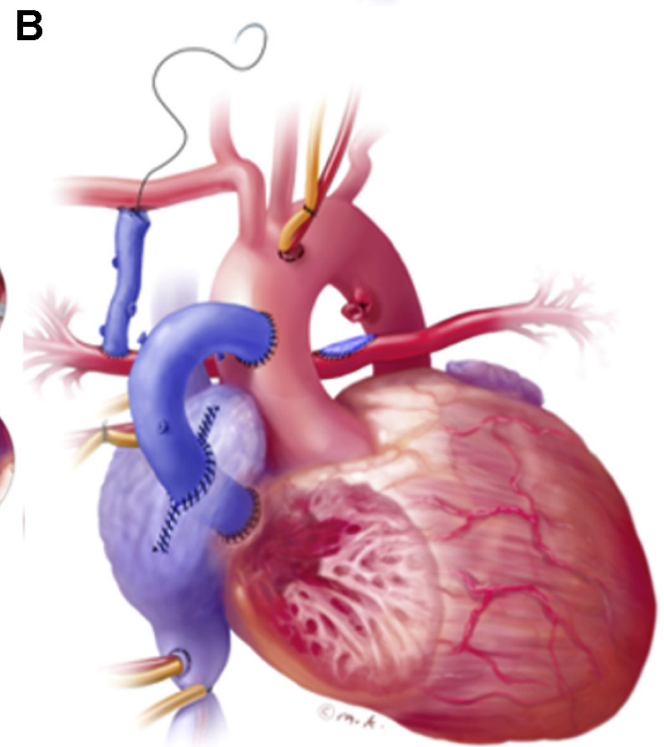

FIGURE 3. Illustrations showing the technical steps of the double shunts. A, Cardiopulmonary bypass is initiated via aortic and bicaval cannulation and cardioplegic arrest is achieved via direct administration of cardioplegia in the right ventricle. B, After cardioplegic arrest, right atriotomy is performed, and the tricuspid valve leaflets are excised (inset). C, The distal graft anastomosis is constructed to the tricuspid valve annulus (inset), then the graft is brought through the right atriotomy, and the proximal anastomosis is constructed to the ascending aorta. Finally, the ductus arteriosus is divided and a modified Blalock-Taussig shunt is created with another cryopreserved saphenous vein homograft.

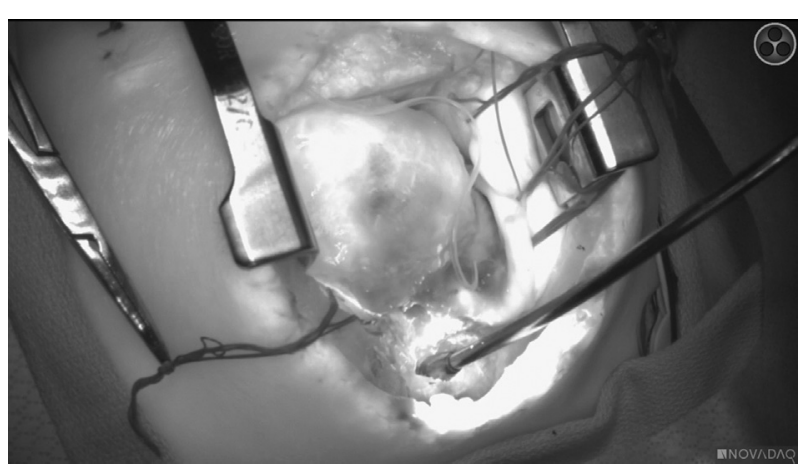

VIDEO 2. Intraoperative fluorescence angiography using indocyanine green injected into the ascending aorta demonstrating widely patent aorto-tricuspid-right ventricular shunt with bidirectional flow in it. Video available at: https://www.jtcvs.org/article/S2666-2507(21)00084-5/ fulltext. steal. She was subsequently weaned off ECMO 48 hours later and her chest was closed 10 days later. Her electrocardiogram readings normalized and troponin levels significantly improved during the postoperative period.

Postoperative echocardiography (Figure 4) and computed tomography scan confirmed patency of the shunt. The patient continued to do well and remained hemodynamically stable until 1 month postoperatively when she started experiencing recurrent episodes of desaturation. She underwent cardiac catheterization to assess the patency of the MBTS because it was left partially clipped after her first surgery. Dilating and stenting of the MBTS was attempted; however, the patient did not tolerate the intervention and her hemodynamic status deteriorated. She was resuscitated and placed on ECMO via the right internal jugular vein and common carotid artery. She was 


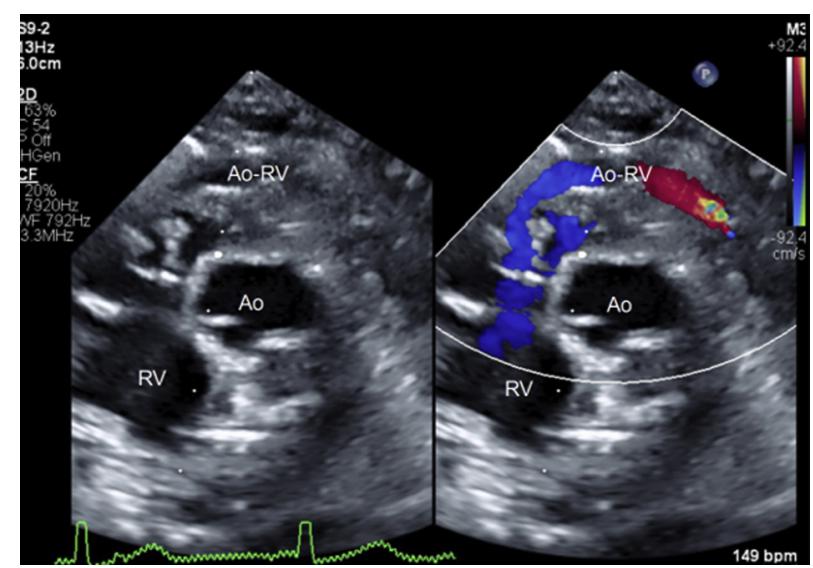

FIGURE 4. Postoperative transthoracic echocardiography demonstrating widely patent aorto-tricuspid-right ventricular shunt. $R V$, Right ventricle; $A o$, aorta; $A o-R V$, aorto-right ventricle shunt.

subsequently taken to an operating room where a repeat median sternotomy was performed and the clips on the MBTS were removed, and she was weaned off ECMO without difficulty. She continued to do well and subsequently underwent transplant 2 months after her initial procedure.

\section{DISCUSSION}

This rare subset of patients with PA is better served with transplantation, and no survival has been demonstrated without transplantation in the presence of bilateral COA (Video 3). The main concern is to stabilize the patient until transplant. With unknown donor availability-especially in neonates-and the related transplant list waiting mortality, in addition to the side effects related to long-term use of prostaglandin therapy, it seems appropriate to consider an alternative strategy.

The initial aim with our current patient was to continue prostaglandin infusion and to wait for an organ, but within the first week of life the baby developed signs of coronary ischemia on echocardiogram and elevation of troponin I

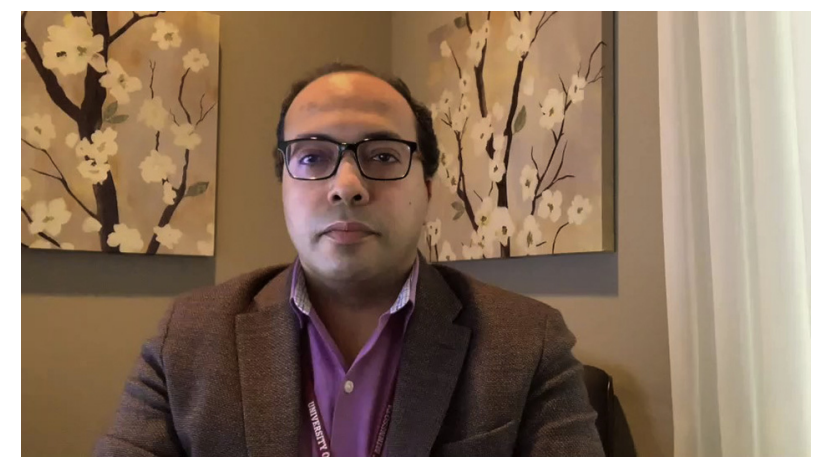

VIDEO 3. Dr Said's discussion of the case with lessons learned and future directions. Video available at: https://www.jtcvs.org/article/S26662507(21)00084-5/fulltext. level when feeds were initiated. Despite intensive care unit admission, holding all enteric nutrition, initiation of total parenteral nutrition, inotropic and pressor support (ie, epinephrine and vasopressin), and delivery of increasing sedation, signs of myocardial ischemia increased. The combination of lower diastolic pressures from the patent ductal arteriosus likely resulted in myocardial steal with continued ST segment elevation, progressive elevation of troponin levels (up to $3.98 \mu \mathrm{g} / \mathrm{L}$ the day before surgery), and markedly elevated $\mathrm{N}$-terminal pro-B-type natriuretic peptide (up to $11,770 \mathrm{pg} / \mathrm{mL}$ preoperatively). The unknown length of time to wait for a donor organ and ongoing progressive signs of ischemia resulted in changing the strategy to provide reliable blood supply to both pulmonary circulation and (more importantly) the myocardium. Although concerns had not been fully de-escalated before transplantation, resolution of troponin levels to near normal occurred $(0.273 \mu \mathrm{g} / \mathrm{L}$ on the day before transplant), feeds had been reinitiated, and she was off all pressors and inotropic supports.

Freeman and colleagues ${ }^{3}$ described the first aorto-RV shunt in 1993 in a 20-month-old patient with Fontan circulation. Success in 5 patients was reported by Laks and colleagues. ${ }^{4}$ All those patients were older, and none had bilateral COA. In a report by Sakurai and colleagues, ${ }^{5}$ the authors performed an aorto-RV shunt in an infant with PA/IVS and bilateral COA who successfully underwent the second-stage palliation with bidirectional cavopulmonary anastomosis.

$\mathrm{RV}$ systolic pressure in these patients is mostly suprasystemic, and the diastolic pressure is lower. Among the concerns during cardiac catheterization of these patients is the inability to estimate the RV end-diastolic pressure (RVEDP) with accuracy due to the catheter-induced tricuspid regurgitation. The same applies for assessment of the degree of tricuspid regurgitation, which can be a bit overestimated. Another concern is that attempts to measure the RV pressure in babies with RVDCC can be life threatening because catheter-induced tricuspid regurgitation can lower the RV systolic pressure resulting in acute myocardial ischemia and significant hemodynamic compromise. We believe/speculate that before intervention the actual RVEDP is not equal to the aortic diastolic pressure because there is no connection to the aorta. If there is a stenosis between the coronary fistula and the RV, one can argue that the RVEDP has to be higher than the left ventricle EDP for blood to flow to the myocardium. After intervention (the aortic-tricuspid RV shunt), we believe RVEDP is lower than the aortic diastolic pressure, which is among the goals of the aortic to RV shunt and we have demonstrated the presence of diastolic flow in the shunt (aortic-to-RV).

We believe that the shunt (in its aorto-RV connection) serves 2 purposes: it decreases the suprasystemic systolic $\mathrm{RV}$ pressure (RV-to-aortic flow), which is favorable to 


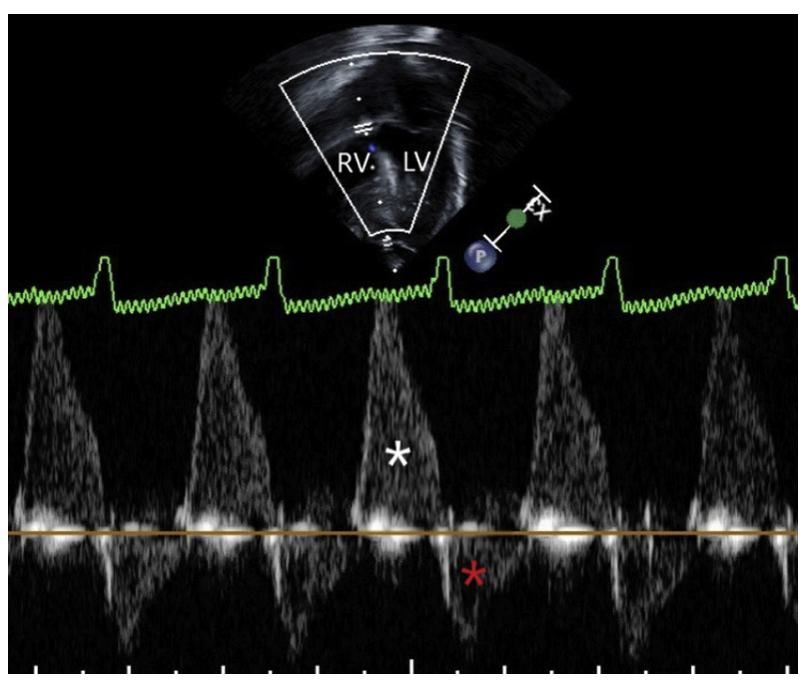

FIGURE 5. Doppler flow showing the bidirectional flow in the aortotricuspid-right ventricular shunt. In diastole (white asterisk), the flow is from the aorta to the right ventricle $(R V)$, whereas in systole (red asterisk) the flow is reversed. $L V$, Left ventricle.

prevent further endocardial/endothelia injury to the RV and related coronary arteries; and in diastole, the flow of the shunt is reversed (aorta-to-RV), which increases the diastolic pressure in the RV to improve coronary blood flow. We have demonstrated the presence of bidirectional flow in the shunt by both intraoperative fluorescence angiography (Video 2) and Doppler echocardiography (Figure 5).

A valid argument may be to place an MBTS/aortopulmonary shunt that is restricted further if necessary to elevate systemic perfusion. This might have been sufficient without taking the additional risk of shunting a suprasystemic RV. However, we believed that an aortopulmonary shunt alone would not be sufficient due to the absence of any natural communication from the aorta to the coronary arterial systems in this baby with bilateral COA, so it was critical to maintain the RVEDP by adding another source of flow to the RV via the aorto-TV-RV shunt. We went to the operating room with already significant elevation in both pro-B-type natriuretic peptide and troponin levels. We believe that an isolated aortopulmonary shunt may have been sufficient if we had at least 1 coronary artery supplied by the native aorta. Additional supportive points are the additional advantages of the aorto-TV-RV shunt; for example, elimination of any tricuspid regurgitation and optimization of oxygen delivery to the RV cavity and to the coronary circulation by elimination of venous influx into the RV. Review of the literature (although in limited reports) for those with bilateral COA supported the use of this additional shunt.

One may argue that the presence of these 2 good-sized shunts may result in overshunting. That was our initial concern because we did not know what the nature of interaction between these 2 shunts and their effects on the patient's hemodynamic parameters would be. Our main goal coming off cardiopulmonary bypass was to maintain adequate coronary perfusion and good diastolic pressure; therefore, we elected to partially reduce the size of the MBTS even farther (by adding hemoclips) and having a bit lower, but tolerable oxygen saturation to maintain good flow in the aorto-to-RV shunt and thus better coronary flow. This worked initially; however, a month later, due to repeat desaturation, we were forced to open the shunt to its original size. We believe the technique works and it may be even more tolerable in larger patients without the need for shunt size reduction initially.

Potential disadvantages of our technique include risk of injury to the conduction tissue that could be similar to TV patch closure, as was described previously, and risk of an RV-to-RA fistula. However, there are several advantages: placing the graft into the TV eliminates any TV regurgitation that has the potential for RV decompression and this increases coronary flow; increasing the amount of oxygenated blood that is delivered into the RV by decreasing venous inflow through the TV; avoiding direct connection to the severely thickened and muscular RV minimizes any potential for anastomotic obstruction; the saphenous vein homografts tend to stretch and this could be another reason to place it to the TV rather than directly to the RV to maintain good control of the shunt flow, especially with the presence of a second shunt (ie, MBTS); and finally using the saphenous vein has been associated with better patency in comparison with any other prosthetic materials. ${ }^{6,7}$

We believe the current technique does not burn any bridges and as we clarify in Video 3, our patient could go either way depending on several factors, including the ability to undergo transplant and availability of an organ. The potential to go down the single-ventricle pathway was described by Sakurai and colleagues. ${ }^{5}$ We believe that our patient, with her current anatomy, is better served with transplant. But we stabilized her to that stage using the current strategy.

\section{CONCLUSIONS}

We propose this double shunt strategy in PA/IVS and RVDCC as an initial stage I palliation for patients with single-ventricle pathway or as a bridge to transplantation.

\section{References}

1. Giglia TM, Mandell VS, Connor AR, Mayer JE, Lock JE. Diagnosis and management of right ventricle-dependent coronary circulation in pulmonary atresia with intact ventricular septum. Circulation. 1992;86:1516-28.

2. Guleserian KJ, Armsby LB, Thiagarajan RR, del Nido PJ, Mayer JE Jr. Natural history of pulmonary atresia with intact ventricular septum and right-ventricledependent coronary circulation managed by the single-ventricle approach. Ann Thorac Surg. 2006;81:2250-7.

3. Freeman JE, DeLeon SY, Lai S, Fisher EA, Ow P, Pifarré R. Right ventricle-toaorta conduit in pulmonary atresia with intact ventricular septum and coronary sinusoids. Ann Thorac Surg. 1993;56:1393-5. 
4. Laks H, Gates RN, Grant PW, Drant S, Allada V, Harake B. Aortic to right ventricular shunt for pulmonary atresia and intact ventricular septum. Ann Thorac Surg. 1995;59:342-7.

5. Sakurai H, Sakurai T, Ohashi N, Nishikawa H. Aortic to right ventricular shunt for pulmonary atresia with intact ventricular septum and bilateral coronary ostial atresia. J Thorac Cardiovasc Surg. 2018;156:e17-20.
6. Tam VK, Murphy K, Parks WJ, Raviele AA, Vincent RN, Strieper M, et al. Saphenous vein homograft: a superior conduit for the systemic arterial shunt in the Norwood operation. Ann Thorac Surg. 2001;71:1537-40.

7. Bogáts G, Kertész E, Katona M, Tószegi A, Kovács GS. Modified Blalock-Taussig shunt using allograft saphenous vein: six years' experience. Ann Thorac Surg. 1996;61:58-62. 